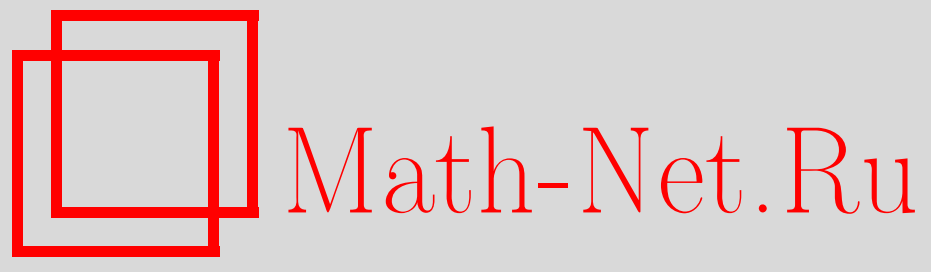

Мейли Лианг, Зонгшенг Гао, О сходимости и росте кратных рядов Дирихле, Матем. заметки, 2010, том 88, выпуск $5,759-769$

DOI: https://doi.org/10.4213/mzm6374

Использование Общероссийского математического портала Math-Net.Ru подразумевает, что вы прочитали и согласны с пользовательским соглашением http://www . mathnet.ru/rus/agreement

Параметры загрузки:

IP : 35.173 .219 .12

26 апреля 2023 г., 08:30:38

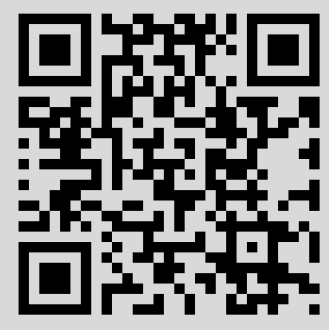


Том 88 выпуск 5 ноябрь 2010

УДК 517.53

\section{О сходимости и росте кратных рядов Дирихле}

\section{Мейли Лианг, Зонгшенг Гао}

В данной работе формула Валирона для простых рядов Дирихле обобщается на $n$-кратные ряды Дирихле и выводится эквивалентное соотношение между порядком $n$-кратного ряда Дирихле, его коэффициентами и показателями.

Библиография: 7 названий.

1. Введение. Рассмотрим $n$-кратный ряд Дирихле

$$
f\left(s_{1}, s_{2}, \ldots, s_{n}\right)=\sum_{m_{1}=1}^{\infty} \sum_{m_{2}=1}^{\infty} \cdots \sum_{m_{n}=1}^{\infty} a_{m_{1} m_{2} \ldots m_{n}} e^{-\lambda_{m_{1}} s_{1}-\lambda_{m_{2}} s_{2}-\cdots-\lambda_{m_{n}} s_{n}},
$$

где для любого $j \in\{1,2, \ldots, n\}$ выполнено $0<\left\{\lambda_{m_{j}}\right\} \uparrow+\infty, s_{j}=\sigma_{j}+i \tau_{j}, \sigma_{j}, \tau_{j} \in \mathbb{R}$.

Чтобы упростить выражение (1.1), введем обозначения $S=\left(s_{1}, s_{2}, \ldots, s_{n}\right) \in \mathbb{C}^{n}$, $m=\left(m_{1}, m_{2}, \ldots, m_{n}\right) \in \mathbb{N}^{n}, \lambda_{m}=\left(\lambda_{m_{1}}, \lambda_{m_{2}}, \ldots, \lambda_{m_{n}}\right) \in \mathbb{R}^{n}$, и

$$
\lambda_{m} S=\left(\lambda_{m_{1}}, \lambda_{m_{2}}, \ldots, \lambda_{m_{n}}\right)\left(s_{1}, s_{2}, \ldots, s_{n}\right)=\lambda_{m_{1}} s_{1}+\lambda_{m_{2}} s_{2}+\cdots+\lambda_{m_{n}} s_{n} .
$$

Таким образом, (1.1) можно также записать в виде

$$
f(S)=\sum_{m} a_{m} e^{-\lambda_{m} S}
$$

Пусть

$$
S_{m}(S)=\sum_{m} a_{j} e^{-\lambda_{j} s}=\sum_{j_{1}=1}^{m_{1}} \sum_{j_{2}=1}^{m_{2}} \cdots \sum_{j_{n}=1}^{m_{n}} a_{j_{1} j_{2} \ldots j_{n}} e^{-\lambda_{j_{1}} s_{1}-\lambda_{j_{2}} s_{2}-\cdots-\lambda_{j_{n}} s_{n}} .
$$

Если множество $\left\{S_{m}(S)\right\}$ ограничено для некоторого множества комплексных чисел $\left(s_{1}, s_{2}, \ldots, s_{n}\right)$ в $\mathbb{C}^{n}$ и существует предел

$$
\lim _{\substack{m_{j} \rightarrow \infty \\ j=1,2, \ldots, n}} S_{m}(S)
$$

то будем говорить, что ряд (1.1) ограниченно сходится для этого множества чисел, и этот предел будем называть суммой ряда (1.1). Если $\left\{S_{m}\left(\sigma_{1_{0}}+i \tau_{1}, \ldots, \sigma_{n_{0}}+i \tau_{n}\right)\right\}$, $m \in \mathbb{N}^{n}$, ограничено равномерно по $-\infty<\tau_{j}<\infty, j=1,2, \ldots, n$, и предел

$$
\lim _{m \rightarrow \infty^{n}} S_{m}\left(\sigma_{1_{0}}+i \tau_{1}, \sigma_{2_{0}}+i \tau_{2}, \ldots, \sigma_{n_{0}}+i \tau_{n}\right)
$$

Работа выполнена при финансовой поддержке Национального научного фонда Китая (грант № 10271011) и Национальной программы основных фундаментальных исследований Китая (грант № 2005СB321902).

(C) МЕйли ЛиАнг, ЗонгшЕнг ГАО, 2010 
существует равномерно по $-\infty<\tau_{j}<\infty, j=1,2, \ldots, n$, то будем говорить, что ряд (1.1) равномерно и ограниченно сходится на $\left(\sigma_{1}=\sigma_{1_{0}}, \sigma_{2}=\sigma_{2_{0}}, \ldots, \sigma_{n}=\sigma_{n_{0}}\right)$. И если предел

$$
\lim _{m \rightarrow \infty^{n}} \sum_{m}\left|a_{j} e^{-\lambda_{j} S}\right|
$$

существует для некоторого множества комплексных чисел $\left(s_{1}, s_{2}, \ldots, s_{n}\right)$, то будем говорить, что ряд (1.1) абсолютно сходится для этого множества чисел. Ряд (1.1) имеет множество соответствующих абсцисс ограниченной сходимости $\left(\sigma_{1_{b}}, \sigma_{2_{b}}, \ldots\right.$, $\left.\sigma_{n_{b}}\right)$, множество соответствующих абсцисс равномерной и ограниченной сходимости $\left(\sigma_{1_{u}}, \sigma_{2_{u}}, \ldots, \sigma_{n_{u}}\right)$, и множество соответствующих абсцисс абсолютной сходимости $\left(\sigma_{1_{a}}, \sigma_{2_{a}}, \ldots, \sigma_{n_{a}}\right)$, где $\sigma_{1_{b}}, \sigma_{2_{b}}, \ldots, \sigma_{n_{b}}, \sigma_{1_{u}}, \sigma_{2_{u}}, \ldots, \sigma_{n_{u}}, \sigma_{1_{a}}, \sigma_{2_{a}}, \ldots, \sigma_{n_{a}}$ могут принимать как конечные, так и бесконечные значения. Если

$$
\sigma_{1}>\sigma_{1_{b}}, \quad \sigma_{2}>\sigma_{2_{b}}, \quad \ldots, \quad \sigma_{n}>\sigma_{n_{b}},
$$

то ряд (1.1) сходится ограниченно, а если

$$
\sigma_{1}<\sigma_{1_{b}}, \quad \sigma_{2}<\sigma_{2_{b}}, \quad \ldots, \sigma_{n}<\sigma_{n_{b}},
$$

то ряд (1.1) не сходится ограниченно. Если

$$
\sigma_{1}=\sigma_{1_{0}}>\sigma_{1_{u}}, \sigma_{2}=\sigma_{2_{0}}>\sigma_{2_{u}}, \quad \ldots, \quad \sigma_{n}=\sigma_{n_{0}}>\sigma_{n_{u}},
$$

то ряд (1.1) равномерно и ограниченно сходится на $\sigma_{1}=\sigma_{1_{0}}, \sigma_{2}=\sigma_{2_{0}}, \ldots, \sigma_{n}=\sigma_{n_{0}}$, а если

$$
\sigma_{1}=\sigma_{1_{1}}<\sigma_{1_{u}}, \quad \sigma_{2}=\sigma_{2_{1}}<\sigma_{2_{u}}, \quad \ldots, \quad \sigma_{n}=\sigma_{n_{1}}<\sigma_{n_{u}},
$$

то ряд (1.1) не сходится равномерно и ограниченно на $\sigma_{1}=\sigma_{1_{1}}, \sigma_{2}=\sigma_{2_{1}}, \ldots$, $\sigma_{n}=\sigma_{n_{1}}$. Если

$$
\sigma_{1}>\sigma_{1_{a}}, \quad \sigma_{2}>\sigma_{2_{a}}, \quad \ldots, \quad \sigma_{n}>\sigma_{n_{a}},
$$

то ряд (1.1) сходится абсолютно, а если

$$
\sigma_{1}<\sigma_{1_{b}}, \quad \sigma_{2}<\sigma_{2_{b}}, \quad \ldots, \quad \sigma_{n}<\sigma_{n_{b}},
$$

то ряд (1.1) не сходится абсолютно.

Построим $n$-мерное пространство чисел $\left\{\sigma_{1}, \sigma_{2}, \ldots, \sigma_{n}\right\}$ и будем обозначать соответствующие абсциссы ограниченной сходимости, соответствующие абсциссы равномерной и ограниченной сходимости, и соответствующие абсциссы абсолютной сходимости точками этого пространства. Теперь мы можем провести прямую линию в этом пространстве следующим образом:

$$
\left\{\begin{array}{l}
\sigma_{1}=r \Phi_{1}(\phi)=r \sin \phi_{n-1} \sin \phi_{n-2} \cdots \sin \phi_{2} \sin \phi_{1}+c_{1}, \\
\sigma_{2}=r \Phi_{2}(\phi)=r \sin \phi_{n-1} \sin \phi_{n-2} \cdots \sin \phi_{2} \cos \phi_{1}+c_{2}, \\
\ldots \ldots \ldots \ldots \ldots \ldots \ldots \ldots \ldots \ldots \ldots \ldots \ldots \ldots \ldots \ldots \ldots \ldots \ldots \ldots \ldots \ldots \ldots \\
\sigma_{n-1}=r \Phi_{n-1}(\phi)=r \sin \phi_{n-1} \cos \phi_{n-2}+c_{n-1}, \\
\sigma_{n}=r \Phi_{n}(\phi)=r \cos \phi_{n-1},
\end{array}\right.
$$

где $\phi=\left(\phi_{1}, \phi_{2}, \ldots, \phi_{n-1}\right) \in(0, \pi / 2)^{n-1}, C=\left(c_{1}, c_{2}, \ldots, c_{n-1}, 0\right) \in \mathbb{R}^{n}$ и $r \in \mathbb{R}-$ переменный параметр. 
Соответствующие абсциссы ограниченной сходимости на прямой обозначим как

$$
\sigma_{b}(C, \phi)=\left(\sigma_{1_{b}}(C, \phi), \sigma_{2_{b}}(C, \phi), \ldots, \sigma_{n_{b}}(C, \phi)\right),
$$

соответствующие абсциссы равномерной и ограниченной сходимости как

$$
\sigma_{u}(C, \phi)=\left(\sigma_{1_{u}}(C, \phi), \sigma_{2_{u}}(C, \phi), \ldots, \sigma_{n_{u}}(C, \phi)\right),
$$

и соответствующие абсциссы абсолютной сходимости как

$$
\sigma_{a}(C, \phi)=\left(\sigma_{1_{a}}(C, \phi), \sigma_{2_{a}}(C, \phi), \ldots, \sigma_{n_{a}}(C, \phi)\right),
$$

которым соответствуют параметры $r_{b}=r_{b}(C, \phi), r_{u}=r_{u}(C, \phi)$ и $r_{a}=r_{a}(C, \phi)$.

При $n=1$ ряд (1.1) является простым рядом Дирихле. Для простых рядов Дирихле получено множество результатов, главным образом касающихся таких объектов как сходимость, рост, распределение значений и т. д. (стандартная библиография дана в [1]-[5]). В работе [2] Валирон получил соотношения, связывающие абсциссы сходимости, абсциссы равномерной сходимости и абсциссы абсолютной сходимости, а также соотношения между этими абсциссами и коэффициентами, каждая из которых называется формулой Валирона. Ю Джиаронг распространил эту формулу на случай двойных радов Дирихле в работе [6]. В данной статье мы обобщим формулу Валирона на случай $n$-кратных рядов Дирихле и докажем соотношение, связывающее порядок $n$-кратного ряда Дирихле и его коэффициенты. В работе [7] Ю Джиаронг изучал сходимость кратных рядов Тайлора. Результаты, полученные в данной статье, обобщают некоторые результаты теории рядов Дирихле. Одновременно они обобщают соответствующие результаты для $n$-кратных рядов Тейлора.

\section{2. Основные результаты.}

Теорема 2.1. Если $\left\{S_{m}(S)\right\}, m \in \mathbb{N}^{n}, S \in \mathbb{C}^{n}$, ограничено в точке $S_{1}=\left(s_{1_{1}}, s_{2_{1}}\right.$, $\left.\ldots, s_{n_{1}}\right) u$

$$
\begin{gathered}
\operatorname{Re}_{1}\left(S_{1}\right)=r_{1} \Phi_{1}(\phi), \quad \operatorname{Re}_{2}\left(S_{1}\right)=r_{1} \Phi_{2}(\phi), \quad \ldots, \quad \operatorname{Re}_{n}\left(S_{1}\right)=r_{1} \Phi_{n}(\phi), \\
\phi=\left(\phi_{1}, \phi_{2}, \ldots, \phi_{n-1}\right) \in(0, \pi / 2)^{n-1}, \text { mo } n p u \\
\operatorname{Re}_{1}\left(S_{2}\right)=r_{2} \Phi_{1}(\phi), \quad \operatorname{Re}_{2}\left(S_{2}\right)=r_{2} \Phi_{2}(\phi), \quad \ldots, \quad \operatorname{Re}_{n}\left(S_{2}\right)=r_{2} \Phi_{n}(\phi),
\end{gathered}
$$

$u r_{2}>r_{1}+D_{\phi}$, ряд (1.1) абсолютно сходится в точке $S_{2}=\left(s_{2_{1}}, s_{2_{2}}, \ldots, s_{n_{2}}\right)$, где

$$
D_{\phi}=\limsup _{|m| \rightarrow \infty} \frac{\log m}{\lambda_{m} \Phi(\phi)}
$$

nрu $m=\left(m_{1}, m_{2}, \ldots, m_{n}\right),|m|=m_{1}+m_{2}+\cdots+m_{n}, \log m=\log \left(m_{1} m_{2} \ldots m_{n}\right)$, $\lambda_{m}=\left(\lambda_{m_{1}}, \lambda_{m_{2}}, \ldots, \lambda_{m_{n}}\right), \Phi(\phi)=\left(\Phi_{1}(\phi), \Phi_{2}(\phi), \ldots, \Phi_{n}(\phi)\right)$,

$$
\lambda_{m} \Phi(\phi)=\lambda_{m_{1}} \Phi_{1}(\phi)+\lambda_{m_{2}} \Phi_{2}(\phi)+\cdots+\lambda_{m_{n}} \Phi_{n}(\phi) .
$$

ДоказАТЕЛьство. Имеем

$$
a_{m} e^{-\lambda_{m} S_{1}}=S_{m_{1} m_{2} \ldots m_{n}}\left(S_{1}\right)-\sum_{1} S_{m}\left(S_{1}\right)+\sum_{2} S_{m}\left(S_{1}\right)-\cdots-(-1)^{n-1} \sum_{n} S_{m}\left(S_{1}\right),
$$


где суммы $\sum_{i} S_{m}\left(S_{1}\right), i=1,2, \ldots, n$, понимаются следующим образом:

$$
\begin{aligned}
& \sum_{1} S_{m}\left(S_{1}\right)=S_{\left(m_{1}-1\right) m_{2} \ldots m_{n}}\left(S_{1}\right) \\
& +S_{m_{1}\left(m_{2}-1\right) m_{3} \ldots m_{n}}\left(S_{1}\right)+\cdots+S_{m_{1} m_{2} \ldots m_{n-1}\left(m_{n}-1\right)}\left(S_{1}\right), \\
& \sum_{2} S_{m}\left(S_{1}\right)=S_{\left(m_{1}-1\right)\left(m_{2}-1\right) \ldots m_{n}}\left(S_{1}\right)+\cdots \\
& +S_{\left(m_{1}-1\right) m_{2} \ldots\left(m_{n}-1\right)}\left(S_{1}\right)+\cdots+S_{m_{1} m_{2} \ldots\left(m_{n-1}-1\right)\left(m_{n}-1\right)}\left(S_{1}\right), \\
& \sum_{n} S_{m}\left(S_{1}\right)=S_{\left(m_{1}-1\right)\left(m_{2}-1\right) \ldots\left(m_{n}-1\right)}\left(S_{1}\right) .
\end{aligned}
$$

Одним словом, $a_{m} e^{-\lambda_{m} S_{1}}$ является алгебраической суммой конечных членов множества $\left\{S_{m}\left(S_{1}\right)\right\}\left(m \in \mathbb{N}^{n}\right) m$. Если же $\left\{S_{m}\left(S_{1}\right)\right\}\left(m \in \mathbb{N}^{n}\right)$ ограничено, то существует предельная константа $K>0$ такая, что

$$
\left|a_{m} e^{-\lambda_{m} S_{1}}\right|<K, \quad m \in \mathbb{N}^{n} .
$$

Пусть $r_{2}-r_{1}>D_{\phi}+\varepsilon, \varepsilon>0$. Имеем

$$
\left|a_{m} e^{-\lambda_{m} S_{2}}\right|=\left|a_{m} e^{-\lambda_{m} S_{1}}\right|\left|a_{m} e^{-\lambda_{m}\left(S_{2}-S_{1}\right)}\right|<K e^{-\left(r_{2}-r_{1}\right) \lambda_{m} \Phi}<K e^{-\left(D_{\phi}+\varepsilon\right) \lambda_{m} \Phi} .
$$

С другой стороны, для некоторого $\varepsilon$, существует положительное целое число $N$ такое, что при $|m|>N$

$$
\frac{\log m}{\lambda_{m} \Phi(\phi)}<D_{\phi}+\frac{\varepsilon}{2} \text {. }
$$

Следовательно,

$$
\left|a_{m} e^{-\lambda_{m} s_{2}}\right|<K e^{-\frac{D_{\phi}+\varepsilon}{D_{\phi}+\frac{\varepsilon}{2}} \log m}=K \cdot\left(m_{1} \cdot m_{2} \cdots m_{n}\right)^{-(1+h)}, \quad h>0 .
$$

Отсюда следует, что

$$
\begin{aligned}
\sum_{|m|=N}^{\infty}\left|a_{m} e^{-\lambda_{m} s_{2}}\right| & \leqslant K \cdot \sum_{|m|=N}^{\infty} \frac{1}{m_{1}^{1+h}} \cdot m_{2}{ }^{1+h} \cdots m_{n}{ }^{1+h} \\
& \leqslant K \cdot \sum_{m_{1}=1}^{\infty} \frac{1}{m_{1}^{1+h}} \cdot \sum_{m_{2}=1}^{\infty} \frac{1}{m_{2}^{1+h}} \cdots \sum_{m_{n}=1}^{\infty} \frac{1}{m_{n}^{1+h}}<+\infty
\end{aligned}
$$

Таким образом, видно, что ряд (1.1) абсолютно сходится на $S_{2}$. Доказательство закончено.

СлЕДСТвиЕ 2.1. Имеем

$$
0 \leqslant r_{a}-r_{b} \leqslant D_{\phi}, \quad \phi \in\left(0, \frac{\pi}{2}\right)^{n-1} .
$$


Tеорема 2.2. Имеем

$$
\limsup _{|m| \rightarrow+\infty} \frac{\log \left|a_{m}\right|-\lambda_{m} C}{\lambda_{m} \Phi(\phi)} \leqslant r_{b} \leqslant r_{u} \leqslant r_{a} \leqslant \limsup _{|m| \rightarrow+\infty} \frac{\log \left|a_{m}\right|-\lambda_{m} C}{\lambda_{m} \Phi(\phi)}+D_{\phi},
$$

где $D_{\phi}$ определено в (2.2).

ДокАЗАтельство. Неравенства $r_{b} \leqslant r_{u} \leqslant r_{a}$ очевидны. Теперь докажем последнее неравенство. Нам нужно рассмотреть только случай $D_{\phi}<+\infty$. Пусть

$$
l=l(C, \phi)=\limsup _{|m| \rightarrow+\infty} \frac{\log \left|a_{m}\right|-\lambda_{m} C}{\lambda_{m} \Phi(\phi)} .
$$

Предположим, что $l$ - ограниченное число. Нужно показать, что если

$\operatorname{Re}_{1}\left(S_{0}\right)=r_{0} \Phi_{1}(\phi)+c_{1}, \quad \ldots, \quad \operatorname{Re}_{(n-1)}\left(S_{0}\right)=r_{0} \Phi_{n-1}(\phi)+c_{n-1}, \quad \operatorname{Re}_{n}\left(S_{0}\right)=r_{0} \Phi_{n}(\phi)$,

$r_{0}>l+D_{\phi}$, то ряд (1.1) сходится абсолютно при $S=S_{0}$.

Пусть $r_{0}=l+D_{\phi}+\varepsilon, \varepsilon>0$. В силу (2.4) при достаточно большом $|m|$ (пусть $\left.|m|>N_{1}\right)$ имеем

$$
\frac{\log \left|a_{m}\right|-\lambda_{m} C}{\lambda_{m} \Phi(\phi)}<l+\frac{\varepsilon}{2}
$$

Одновременно имеем

$$
\begin{aligned}
\left|a_{m} e^{-\lambda_{m} S_{0}}\right| & =\left|a_{m}\right| e^{-r_{0} \lambda_{m} \Phi(\phi)-\lambda_{m} C} \\
& <e^{(l+\varepsilon / 2) \lambda_{m} \Phi(\phi)-\left(l+D_{\phi}+\varepsilon\right) \lambda_{m} \Phi(\phi)}=e^{-\left(D_{\phi}+\varepsilon / 2\right) \lambda_{m} \Phi(\phi)} .
\end{aligned}
$$

С другой стороны, при достаточно большом $|m|$ (пусть $|m|>N_{2}$ ) из определения $D_{\phi}$ немедленно следует, что

$$
\frac{\log m}{\lambda_{m} \Phi(\phi)}<D_{\phi}+\frac{\varepsilon}{4}
$$

Так, при $|m|>\max \left\{N_{1}, N_{2}\right\}$ имеем

$$
\left|a_{m} e^{-\lambda_{m} S_{0}}\right|<e^{-\left(D_{\phi}+\varepsilon / 2\right) /\left(D_{\phi}+\varepsilon / 4\right) \cdot \log m} .
$$

Аналогично последнему этапу в доказательстве теоремы 2.1 легко видеть, что ряд (1.1) абсолютно сходится в $S_{0}$, что доказывает наше утверждение в случае $l<+\infty$. Кроме того, последнее неравенство в (2.3) также выполняется в случае $l=+\infty$.

Далее приведем доказательство первого неравенства в (2.3). Предположим, что если

$$
\operatorname{Re}_{1}\left(S_{1}\right)=r_{1} \Phi_{1}(\phi)+c_{1}, \quad \ldots, \quad \operatorname{Re}_{(n-1)}\left(S_{1}\right)=r_{1} \Phi_{n-1}(\phi)+c_{n-1}, \quad \operatorname{Re}_{n}\left(S_{1}\right)=r_{1} \Phi_{n},
$$

то ряд (1.1) ограниченно сходится в $S=S_{1}$. Тогда, так же как и в начале доказательства теоремы 2.1, существует ограниченная константа $K>0$ такая, что

$$
\left|a_{m} e^{-\lambda_{m} S_{1}}\right|<K, \quad m \in \mathbb{N}^{n} .
$$

Так как

$$
a_{m}=a_{m} e^{-\lambda_{m} S_{1}} \cdot e^{\lambda_{m} S_{1}}
$$


имеем

$$
\left|a_{m}\right|<K \cdot\left|e^{\lambda_{m} S_{1}}\right|=K \cdot e^{\lambda_{m} C} e^{r_{1}\left(\lambda_{m} \Phi(\phi)\right)} .
$$

Проводя простые выкладки и вычисляя limsup, получим

$$
l=\limsup _{|m| \rightarrow+\infty} \frac{\log \left|a_{n}\right|-\lambda_{m} C}{\lambda_{m} \Phi(\phi)} \leqslant r_{1} .
$$

Следовательно, если $l=-\infty$ и $l$ ограничено, то выполняется первое неравенство в (2.3).

Для завершения доказательства надо рассмотреть случай $l=+\infty$. В этом случае для любых положительных ограниченных чисел $r_{2}$ и $K_{1}$ можно получить следующее неравенство:

$$
l=\limsup _{|m| \rightarrow+\infty} \frac{\log \left|a_{n}\right|-\lambda_{m} C}{\lambda_{m} \Phi(\phi)}>r_{2}+K_{1}
$$

Так можно найти $n$ рядов, состоящих из целых чисел $\left\{m_{j_{i}}\right\}, j=1,2, \ldots, n, i=$ $1,2,3, \ldots$, и таких, что $\left|m_{i}\right|=m_{1_{i}}+m_{2_{i}}+\cdots+m_{n_{i}} \rightarrow+\infty$ и

$$
\left|a_{m_{i}}\right| e^{-\lambda_{m_{i}} C} \geqslant e^{\left(r_{2}+K_{1}\right) \lambda_{m_{i}} \Phi(\phi)} .
$$

Это значит, что

$$
\left|a_{m_{i}} e^{-\lambda_{m_{i}} S_{2}}\right| \geqslant e^{K_{1} \lambda_{m_{i}} \Phi(\phi)},
$$

откуда следует, что $\left\{a_{m} e^{-\lambda_{m} S_{2}}\right\}, m \in \mathbb{N}^{n}$, ограничено. Итак, $\left\{S_{m}(S)\right\}, m \in \mathbb{N}^{n}$, ограничено, а ряд (1.1) не сходится ограниченно в $S_{2}$, что завершает доказательство.

Теорема 2.3. Для ряда (1.1), если $\sigma_{j_{u}}<+\infty, j=1,2, \ldots, n$, то для любого произвольного $\sigma_{j}>\sigma_{j_{u}}$ и любого произвольного $m_{j} \in \mathbb{N}, j=1,2, \ldots, n$, выполняется соотношение

$$
a_{m} e^{-\lambda_{m} \sigma}=\lim _{\substack{T_{j} \rightarrow+\infty \\ j=1,2, \ldots, n}} \frac{1}{\prod_{j} T_{j}} \int_{t_{1}}^{T_{1}} \int_{t_{2}}^{T_{2}} \cdots \int_{t_{n}}^{T_{n}} f\left(S_{1}\right) e^{i \lambda_{m} \tau} d \tau_{1} d \tau_{2} \ldots d \tau_{n},
$$

где $\lambda_{m}=\left(\lambda_{m_{1}}, \lambda_{m_{2}} \ldots, \lambda_{m_{n}}\right) \in \mathbb{R}^{n}, \sigma=\left(\sigma_{1}, \sigma_{2}, \ldots, \sigma_{n}\right) \in \mathbb{R}^{n}, \tau=\left(\tau_{1}, \tau_{2}, \ldots, \tau_{n}\right) \in \mathbb{R}^{n}$,

$$
S_{1}=\left(s_{1_{1}}, s_{2_{1}}, \ldots, s_{n_{1}}\right)=\left(\sigma_{1_{1}}+i \tau_{1_{1}}, \sigma_{2_{1}}+i \tau_{2_{1}}, \ldots, \sigma_{n_{1}}+i \tau_{n_{1}}\right) \in \mathbb{C}^{n},
$$

$\sigma_{j_{1}}, \tau_{j_{1}} \in \mathbb{R}, j_{1}=1,2, \ldots, n, u t_{j}, j=1,2, \ldots, n,-$ произвольные фиксированные действительные числа.

ДокАЗАТЕЛЬСТво. Если $m_{j_{0}} \neq m_{j_{1}}, j=1,2, \ldots, n$, то

$$
\lim _{\substack{T_{j} \rightarrow+\infty \\ j=1,2, \ldots, n}} \frac{1}{\prod_{j} T_{j}} \int_{t_{1}}^{T_{1}} \int_{t_{2}}^{T_{2}} \cdots \int_{t_{n}}^{T_{n}} e^{i\left(\lambda_{m}^{0}-\lambda_{m}^{1}\right) \tau} d \tau_{1} d \tau_{2} \ldots d \tau_{n}=0
$$


где $\lambda_{m}^{0}=\left(\lambda_{m_{1_{0}}}, \lambda_{m_{2_{0}}}, \ldots, \lambda_{m_{n_{0}}}\right), \lambda_{m}^{1}=\left(\lambda_{m_{1_{1}}}, \lambda_{m_{2_{1}}}, \ldots, \lambda_{m_{n_{1}}}\right)$. Действительно,

$$
\begin{aligned}
\lim _{\substack{T_{j} \rightarrow+\infty \\
j=1,2, \ldots, n}} \frac{1}{\prod_{j} T_{j}} \int_{t_{1}}^{T_{1}} e^{i\left(\lambda_{m_{1}}-\lambda_{m_{1}}\right) \tau_{1}} d \tau_{1} \\
\times \int_{t_{2}}^{T_{2}} e^{i\left(\lambda_{m_{2}}-\lambda_{m_{2}}\right) \tau_{2}} d \tau_{2} \cdots \int_{t_{n}}^{T_{n}} e^{i\left(\lambda_{m_{n_{0}}}-\lambda_{m_{n_{1}}}\right) \tau_{n}} d \tau_{n} \\
=\lim _{\substack{T_{j} \rightarrow+\infty \\
j=1,2, \ldots, n}} \frac{1}{\prod_{j} T_{j}}\left[\left.\left.\frac{e^{i\left(\lambda_{m_{1}}-\lambda_{m_{1}}\right) \tau_{1}}}{i\left(\lambda_{m_{1_{0}}}-\lambda_{m_{1}}\right)}\right|_{t_{1}} ^{T_{1}} \ldots \frac{e^{i\left(\lambda_{m_{n_{0}}}-\lambda_{m_{n_{1}}}\right) \tau_{n}}}{i\left(\lambda_{m_{n_{0}}}-\lambda_{m_{n_{1}}}\right)}\right|_{t_{n}} ^{T_{n}}\right]=0 .
\end{aligned}
$$

Теперь положим $\sigma_{1}>\sigma_{1_{u}}, \sigma_{2}>\sigma_{2_{u}}, \ldots, \sigma_{n}>\sigma_{n_{u}}$. Тогда

$$
\begin{aligned}
& \frac{1}{\prod_{j} T_{j}} \int_{t_{1}}^{T_{1}} \int_{t_{2}}^{T_{2}} \ldots \int_{t_{n}}^{T_{n}} f(S) e^{\lambda_{m}^{1} S} d \tau_{1} d \tau_{2} \ldots d \tau_{n} \\
& \quad=\frac{1}{\prod_{j} T_{j}} \int_{t_{1}}^{T_{1}} \int_{t_{2}}^{T_{2}} \cdots \int_{t_{n}}^{T_{n}} \sum_{m} a_{m} e^{\left(\lambda_{m}-\lambda_{m}^{1}\right) S} d \tau_{1} d \tau_{2} \ldots d \tau_{n} .
\end{aligned}
$$

Покажем, что интеграл существует. Разделим каждое $m_{j} \in \mathbb{N}^{+}, j=1,2, \ldots, n$, на три части, именно, $1 \leqslant m_{j} \leqslant m_{j_{1}}, m_{j}=m_{j_{1}}$ и $m_{j_{1}}+1 \leqslant m_{j}<\infty$. Тогда вышеупомянутый интеграл можно записать как сумму $3^{n}$ членов, каждый из которых ограничен. Следовательно, если $T_{j} \rightarrow+\infty, j=1,2, \ldots, n$, то каждый член стремится к нулю, за исключением одного члена, где $m_{j}=m_{j_{1}}$ для всех $j=1,2, \ldots, n$; предел этого члена равен $a_{m}$. Таким образом, уравнение (2.5) выполняется.

СлЕДСТВиЕ 2.2. Для ряда (1.1)

$$
m(\sigma, f) \leqslant M(\sigma, f) .
$$

Здесь

$$
m(\sigma, f)=m\left(\sigma_{1}, \sigma_{2}, \ldots, \sigma_{n}, f\right)=\max _{m \in \mathbb{N}^{n}}\left\{\left|a_{m}\right| e^{-\lambda_{m} \sigma}\right\}
$$

называется максимальным членом п-кратного ряда Дирихле, а

$$
\begin{aligned}
M(\sigma, f) & =M\left(\sigma_{1}, \sigma_{2}, \ldots, \sigma_{n}, f\right) \\
& =\sup \left\{\left|f\left(\sigma_{1}+i \tau_{1}, \sigma_{2}+i \tau_{2}, \ldots, \sigma_{n}+i \tau_{n}\right)\right|:-\infty<\tau_{j}<+\infty, j=1,2, \ldots, n\right\},
\end{aligned}
$$

называется максимальным модулем п-кратного ряда Дирихле.

Предположим, что для любого фиксированного $\phi \in(0, \pi / 2)^{n-1}$

$$
\begin{gathered}
l=l(c, \phi)=\varlimsup_{|m| \rightarrow+\infty} \frac{\log \left|a_{m}\right|-\lambda_{m} C}{\lambda_{m} \Phi}=0, \\
\limsup _{m_{j} \rightarrow+\infty} \frac{m_{j}}{\lambda_{m_{j}}}=0, \quad j=1,2, \ldots, n .
\end{gathered}
$$

Из (2.7) следует, что $D_{\phi}=0$. Это значит, что $r_{b}=r_{u}=r_{a}=0$, т.е.

$$
\begin{gathered}
\sigma_{b_{1}}=\sigma_{u_{1}}=\sigma_{a_{1}}=c_{1}, \quad \ldots, \quad \sigma_{b_{(n-1)}}=\sigma_{u_{(n-1)}}=\sigma_{a_{(n-1)}}=c_{(n-1)}, \\
\sigma_{b_{n}}=\sigma_{u_{n}}=\sigma_{a_{n}}=0 .
\end{gathered}
$$

В дальнейшем будем полагать, что $c=0$. В противном случае нужно применить только преобразование переноса. 
ЛЕмма 2.1. Если выполнены условия (2.6) и (2.7), при любом $\varepsilon>0$ и любом $\sigma_{j}>0, j=1,2, \ldots, n$, яяд (1.1) имеет следующее свойство:

$$
m(\sigma, f) \leqslant M(\sigma, f) \leqslant K(\varepsilon) \frac{m((1-\varepsilon) \sigma, f)}{\Pi_{j=1}^{n} \sigma_{j}},
$$

где $K(\varepsilon)$ - константа относителъно $\varepsilon$ и $f(S)$.

ДокАзАТЕЛЬСтво. Первое неравенство выводится из следствия 2.2, и нам остается доказать только второе неравенство.

В силу $(2.7)$ для произвольного $\varepsilon>0$ существуют $N_{j}=N_{j}(\varepsilon)$ такие, что

$$
m_{j}<\left(D_{j}+\varepsilon\right) \lambda_{m_{j}} \leqslant(D+\varepsilon) \lambda_{m_{j}}, \quad m_{j}>N_{j},
$$

где $D=\max \left\{D_{j}, j=1,2, \ldots, n\right\}$.

Выберем $\sigma_{j}>0, j=1,2, \ldots, n$, и получим

$$
\begin{aligned}
M(\sigma, f) & \leqslant \sum_{|m|=n}^{N_{0}}\left|a_{m}\right| e^{-\lambda_{m} \sigma}+\sum_{|m|=N_{0}+1}^{+\infty}\left|a_{m}\right| e^{-\lambda_{m} \sigma} \\
& =\sum_{|m|=n}^{N_{0}}\left|a_{m}\right| e^{-\lambda_{m} \sigma}+\sum_{|m|=N_{0}+1}^{+\infty}\left|a_{m}\right| e^{-\lambda_{m}(1-\varepsilon) \sigma} \cdot e^{-\lambda_{m} \varepsilon \sigma} \\
& \leqslant K_{0} m(\sigma, f)+m((1-\varepsilon) \sigma) \cdot \prod_{j=1}^{n} \frac{1}{1-e^{-\varepsilon \sigma_{j} /(D+\varepsilon)}},
\end{aligned}
$$

где $K_{0}=\sum_{|m|=n}^{N_{0}} 1=\left(\begin{array}{c}N_{0} \\ n\end{array}\right)$. Из этого неравенства следует искомое утверждение.

ОПРЕДЕЛЕНИЕ 2.1. Если выполняются условия (2.6) и (2.7) и если

$$
\limsup _{\substack{\sigma_{j} \rightarrow 0^{+} \\ j=1,2, \ldots, n}} \frac{\log ^{+} \log ^{+} M(\sigma, f)}{\log \left(1 / \sigma_{1}+1 / \sigma_{2}+\cdots+1 / \sigma_{n}\right)}=\rho,
$$

то будем говорить, что $f(S)$ имеет порядок $\rho$ в области $\sigma_{j}>0, j=1,2, \ldots, n$, где $\log ^{+} x=\max \{\log x, 0\}$.

ЛЕмма 2.2. В условиях леммы 2.1,

$$
\limsup _{\substack{\sigma_{j} \rightarrow 0^{+} \\ j=1,2, \ldots, n}} \frac{\log ^{+} \log ^{+} m(\sigma, f)}{\log \left(1 / \sigma_{1}+1 / \sigma_{2}+\cdots+1 / \sigma_{n}\right)}=\rho .
$$

Лемма 2.3. Пусть $c \in(0,1) u \sigma_{j}>0, j=1,2, \ldots, n,-$ константы. Тогда функиия

$$
\phi\left(x_{1}, x_{2}, \ldots, x_{n}\right)=x_{1}^{c}+x_{2}^{c}+\cdots+x_{n}^{c}-x_{1} \sigma_{1}-x_{2} \sigma_{2}-\cdots-x_{n} \sigma_{n},
$$

$x_{j}>0, j=1,2, \ldots, n$, достигает максимума

$$
(1-c)\left[\left(\frac{c}{\sigma_{1}}\right)^{c /(1-c)}+\left(\frac{c}{\sigma_{2}}\right)^{c /(1-c)}+\cdots+\left(\frac{c}{\sigma_{n}}\right)^{c /(1-c)}\right]
$$


в точке

$$
\left(x_{1}, x_{2}, \ldots, x_{n}\right)=\left(\left(\frac{c}{\sigma_{1}}\right)^{1 /(1-c)},\left(\frac{c}{\sigma_{2}}\right)^{1 /(1-c)}, \ldots,\left(\frac{c}{\sigma_{n}}\right)^{1 /(1-c)}\right) .
$$

Лемма 2.4. Пусть ь и $x_{1}, x_{2}, \ldots, x_{n}$ - положстелънъе константъ. Тогда функиця

$$
\varphi\left(\sigma_{1}, \sigma_{2}, \ldots, \sigma_{n}\right)=\sigma_{1}^{-c}+\sigma_{2}^{-c}+\cdots+\sigma_{n}^{-c}+x_{1} \sigma_{1}+x_{2} \sigma_{2}+\cdots+x_{n} \sigma_{n},
$$

$\sigma_{j}>0, j=1,2, \ldots, n$, достигает минимума

в точке

$$
(1+c)\left[\left(\frac{x_{1}}{c}\right)^{c /(1+c)}+\left(\frac{x_{2}}{c}\right)^{c /(1+c)}+\cdots+\left(\frac{x_{n}}{c}\right)^{c /(1+c)}\right]
$$

$$
\left(\sigma_{1}, \sigma_{2}, \ldots, \sigma_{n}\right)=\left(\left(\frac{c}{x_{1}}\right)^{1 /(1+c)},\left(\frac{c}{x_{2}}\right)^{1 /(1+c)}, \ldots,\left(\frac{c}{x_{n}}\right)^{1 /(1+c)}\right)
$$

Теорема 2.4. Предположим, что ряд (1.1) удовлетворяет условиям (2.6), (2.7). Тогда в области $\operatorname{Re}\left(s_{1}\right)>0, \operatorname{Re}\left(s_{2}\right)>0, \ldots, \operatorname{Re}\left(s_{n}\right)>0$

$f(S)$ имеет порядок $\rho \Longleftrightarrow \limsup _{|m| \rightarrow+\infty} \frac{\log ^{+} \log ^{+}\left|a_{m}\right|}{\log \left(\sum_{j=1}^{n} \lambda_{m_{j}}\right)}= \begin{cases}\frac{\rho}{\rho+1}, & \text { если } \rho<+\infty, \\ 1, & \text { если } \rho=+\infty .\end{cases}$

ДокАзАтельство. Сначала рассмотрим случай $\rho<+\infty$.

Теперь надо доказать, что правая часть предыдущей формулы является необходимым условием для того, чтобы $f(S)$ имела порядок $\rho$ в области $\operatorname{Re}\left(s_{j}\right)>0$, $j=1,2, \ldots, n$.

В силу леммы 2.2 для произвольных достаточно малых $\sigma_{j}>0, j=1,2, \ldots, n$, и для любых $m_{j} \in \mathbb{N}, j=1,2, \ldots, n$, имеем

$$
\left|a_{m}\right| e^{-\lambda_{m} \sigma} \leqslant m(\sigma, f)<\exp \left\{\left(\frac{1}{\sigma_{1}}+\frac{1}{\sigma_{2}}+\cdots+\frac{1}{\sigma_{n}}\right)^{\rho+\varepsilon}\right\}
$$

так что

$$
\begin{aligned}
\log \left|a_{m}\right| & <\left(\frac{1}{\sigma_{1}}+\frac{1}{\sigma_{2}}+\cdots+\frac{1}{\sigma_{n}}\right)^{\rho+\varepsilon}+\lambda_{m_{1}} \sigma_{1}+\lambda_{m_{2}} \sigma_{2}+\cdots+\lambda_{m_{n}} \sigma_{n} \\
& <n^{(\rho+\varepsilon)}\left(\sigma_{1}^{-(\rho+\varepsilon)}+\sigma_{2}^{-(\rho+\varepsilon)} \cdots+\sigma_{n}^{-(\rho+\varepsilon)}\right)+\lambda_{m_{1}} \sigma_{1}+\lambda_{m_{2}} \sigma_{2}+\cdots+\lambda_{m_{n}} \sigma_{n} .
\end{aligned}
$$

Таким образом, для достаточно больших $m_{j}, j=1,2, \ldots, n$, если мы возьмем

$$
\sigma_{1}=\left(\frac{\rho+\varepsilon}{\lambda_{m_{1}}}\right)^{1 /(\rho+\varepsilon+1)}, \quad \sigma_{2}=\left(\frac{\rho+\varepsilon}{\lambda_{m_{2}}}\right)^{1 /(\rho+\varepsilon+1)}, \ldots, \quad \sigma_{n}=\left(\frac{\rho+\varepsilon}{\lambda_{m_{n}}}\right)^{1 /(\rho+\varepsilon+1)},
$$

то в силу леммы 2.4 получим

$$
\begin{aligned}
\log \left|a_{m}\right| & <n^{\rho+\varepsilon}(\rho+\varepsilon+1)\left[\left(\frac{\lambda_{m_{1}}}{\rho+\varepsilon}\right)^{(\rho+\varepsilon) /(\rho+\varepsilon+1)}\right. \\
& \left.\quad+\left(\frac{\lambda_{m_{2}}}{\rho+\varepsilon}\right)^{(\rho+\varepsilon) /(\rho+\varepsilon+1)}+\cdots+\left(\frac{\lambda_{m_{n}}}{\rho+\varepsilon}\right)^{(\rho+\varepsilon) /(\rho+\varepsilon+1)}\right] \\
& <n^{\rho+\varepsilon+1}(\rho+\varepsilon+1)\left[\frac{\lambda_{m_{1}}}{\rho+\varepsilon}+\frac{\lambda_{m_{2}}}{\rho+\varepsilon}+\cdots+\frac{\lambda_{m_{n}}}{\rho+\varepsilon}\right]^{(\rho+\varepsilon) /(\rho+\varepsilon+1)} .
\end{aligned}
$$


Следовательно,

$$
\limsup _{|m| \rightarrow+\infty} \frac{\log ^{+} \log ^{+}\left|a_{m}\right|}{\log \left(\sum_{j=1}^{n} \lambda_{m_{j}}\right)} \leqslant \frac{\rho+\varepsilon}{\rho+\varepsilon+1} .
$$

Так как $\varepsilon$ выбран произвольным образом, имеем

$$
\limsup _{|m| \rightarrow+\infty} \frac{\log ^{+} \log ^{+}\left|a_{m}\right|}{\log \left(\sum_{j=1}^{n} \lambda_{m_{j}}\right)} \leqslant \frac{\rho}{\rho+1} .
$$

Предположим, что неравенство в предыдущей формуле сохраняет знак. Тогда $\rho>0$ и существует $\delta \in(0, \rho)$ такая, что

$$
\limsup _{|m| \rightarrow+\infty} \frac{\log ^{+} \log ^{+}\left|a_{m}\right|}{\log \left(\sum_{j=1}^{n} \lambda_{m_{j}}\right)} \leqslant \frac{\rho-\delta}{\rho-\delta+1} .
$$

Следовательно, при достаточно большом $|m|$ в силу леммы 2.3 имеем

$$
\begin{aligned}
\log \left|a_{m}\right| e^{-\lambda_{m} \sigma} & \leqslant\left(\sum_{j=1}^{n} \lambda_{m_{j}}\right)^{(\rho-\delta) /(\rho-\delta+1)}-\lambda_{m} \sigma \\
& \leqslant \lambda_{m_{1}}^{(\rho-\delta) /(\rho-\delta+1)}+\lambda_{m_{2}}^{(\rho-\delta) /(\rho-\delta+1)}+\cdots+\lambda_{m_{n}}^{(\rho-\delta) /(\rho-\delta+1)}-\lambda_{m} \sigma \\
& \leqslant \frac{1}{\rho-\delta+1}\left[\left(\frac{1}{\sigma_{1}} \frac{\rho-\delta}{\rho-\delta+1}\right)^{\rho-\delta}+\left(\frac{1}{\sigma_{2}} \frac{\rho-\delta}{\rho-\delta+1}\right)^{\rho-\delta}+\cdots\right. \\
& \left.\leqslant \frac{1}{\rho-\delta+1}\left[\left(\frac{1}{\sigma_{n}} \frac{\rho-\delta}{\rho-\delta+1}\right)^{\rho-\delta}\right]+\left(\frac{1}{\sigma_{2}}\right)^{\rho-\delta}+\cdots+\left(\frac{1}{\sigma_{n}}\right)^{\rho-\delta}\right] \\
& \leqslant \frac{n}{\rho-\delta+1}\left(\frac{1}{\sigma_{1}}+\frac{1}{\sigma_{2}}+\cdots+\frac{1}{\sigma_{n}}\right)^{\rho-\delta} \cdot
\end{aligned}
$$

Таким образом,

$$
\limsup _{\substack{\sigma_{j} \rightarrow 0^{+} \\ j=1,2, \ldots, n}} \frac{\log ^{+} \log ^{+} m(\sigma, f)}{\log \left(1 / \sigma_{1}+1 / \sigma_{2}+\cdots+1 / \sigma_{n}\right)} \leqslant \rho-\delta<\rho,
$$

что противоречит предположению. Следовательно, в случае $\rho<\infty$ соотношение

$$
\limsup _{|m| \rightarrow+\infty} \frac{\log ^{+} \log ^{+}\left|a_{m}\right|}{\log \left(\sum_{j=1}^{n} \lambda_{m_{j}}\right)}=\frac{\rho}{\rho+1}
$$

является необходимым условием для того, чтобы $f(S)$ имела порядок $\rho$ в области $\operatorname{Re}\left(s_{1}\right)>0, \operatorname{Re}\left(s_{2}\right)>0, \ldots, \operatorname{Re}\left(s_{n}\right)>0$.

Применяя леммы 2.3 и 2.4, можно доказать, что (2.9) является и достаточны условием.

Доказательство в случае $\rho=\infty$ аналогично. Таким образом, теорема полностью доказана.

Авторы выражают искреннюю благодарность проф. Сун Даочун за помощь и ценные советы. 


\section{СПИСОК ЦИТИРОВАННОЙ ЛИТЕРАТУРЫ}

[1] Daochun Sun, Jiarong Yu, "Sur la distribution des valeurs de certaines séries aléatoires de Dirichlet. II", C. R. Acad. Sci. Paris Sér. I Math., 1989:7 (308), 205-207.

[2] G. Valiron, "Entire functions and Borel's directions", Proc. Natl. Acad. Sci. USA, 20:3 (1934), 211-215.

[3] Chia-Yung Yu, "Sur la croissance de certaines séries de Dirichlet sur des demi-droites horizontales", C. R. Acad. Sci. Paris Sér. I Math., 296:4 (1983), 187-190.

[4] Jia-rong Yu, "Julia lines of random Dirichlet series", Bull. Sci. Math., 128:5 (2004), 341-353.

[5] Jiarong $\mathrm{Yu}$, "On the growth and the distribution of values of exponential series convergent only in the right half-plane", Chinese Ann. Math., 3:4 (1982), 545-554.

[6] Yu Chia-yung, "On the convergence of double Dirichet series and double Laplace transform", J. Wuhan Univ. (Natural Sc. Ed.), 1:1 (1962), 1-16 (in Chinese).

[7] Yu Jiarong, "Some properties of multiple Taylor series and random Taylor series", Acta Math. Sci. Ser. B Engl. Ed., 26:3 (2006), 568-576.

Мейли Лианг

Поступило

Beihang University, Beijing, Китай

28.08 .2009

E-mail: liangmeili2004@163.com

Зонгшенг Гао

Beihang University, Beijing, Китай

E-mail: zshgao@buaa.edu.cn 